\title{
Demand for measles and yellow fever vaccines for children in Ghana: are socio-economic, demographic and Geographic factors relevant?
}

\author{
Mustapha Immurana ${ }^{1 *}$, Arabi Urma ${ }^{2}$ \\ ${ }^{1}$ Research Scholar, Department of Economics, Mangalore University, Mangalagangotri 574119, Karnataka State, India \\ ${ }^{2}$ Professor and Research Guide, Department of Economics, Mangalore University, Mangalagangotri 574119, Karnataka State, India \\ *Corresponding author E-mail: mustaphaimmurana@gmail.com
}

\begin{abstract}
One of the best ways of Preventing Measles and Yellow fever which are dangerous killers of children is through vaccination. Therefore given the absence of research to the best of our knowledge on the factors that affect demand for Measles and Yellow fever vaccines among children in Ghana, this study investigated the socio-economic, demographic and geographic factors that affect the demand for Measles and Yellow fever vaccines among children in Ghana. By using data from the 2014 Ghana Demographic and Health Survey and the binary probit model, the study among other findings revealed that, Children in the Western region were less likely to have received both the Measles 1 and Yellow fever vaccines. Also urban children and children with unemployed mothers were found to be less likely to have received the Measles 1, Measles 2 and Yellow fever vaccines relative to rural children and children with employed mothers respectively. Also Traditional/Spiritualist/No religion faith children were found to be less likely to have received the Yellow fever vaccine. Further, uneducated mothers, mothers without health insurance and non-wealthy households were found to be less likely to demand the Measles 2 vaccine for their children. This study therefore concludes that Socio-Economic, Demographic and Geographic Factors are relevant determinants of demand for measles and yellow fever vaccines among children in Ghana.
\end{abstract}

Keywords: Demand for Health; Child Health; Immunisation; Measles Vaccine; Yellow Fever Vaccine; Ghana.

\section{Introduction}

Irrespective of the existence of a cost-effective and safe vaccine for measles, it is regarded as one of the major causes of mortalities in young children. Globally, measles killed 114900 (about thirteen (13) deaths each hour) people mostly children underfive in 2014. In 1980 prior to the existence of widespread measles vaccination, it was approximated that measles was responsible for 2.6 million deaths every year. However using measles vaccination, it is estimated that 17.1 million mortalities or deaths were prevented from 2000 to 2014. It is further stated that, unvaccinated children have the highest risk to contract measles and its accompanying complications which includes death (World Health Organisation [WHO], 2016a). Yellow fever is also transmitted by infected mosquitoes and the virus that causes it is endemic in the tropical areas of Africa and South and Central America (WHO, 2016b). The past two decades have experienced an increase in the number of yellow fever cases due to urbanisation, deforestation, climate change, falling population immunity to infection and population movements. Yellow fever is responsible for 30,000 deaths worldwide out of 200,000 cases yearly and $90 \%$ of these deaths occur in Africa. Further, without treatment, $50 \%$ of persons severely affected with yellow fever will die. However, the most preventable means with regards to Yellow fever is using the Yellow fever vaccine which is effective and less costly (WHO, 2014).

As part of the Immunization Programme in Ghana, Children are supposed to receive one dose of the yellow fever vaccine and two doses of the measles vaccine. Thus a measles rubella-vaccine giv- en at nine months which was introduced in 2013 to replace the measles-only vaccine at nine months and a second dose measles vaccine which was introduced in 2012 as a booster dose to be given at eighteen months (Ghana Statistical Service [GSS], Ghana Health Service [GHS] \& ICF International, 2015). Thus given the deadly nature of measles and yellow fever to child survival coupled with the fact that Ghana's underfive mortality rate of sixty deaths per thousand live births as reported by GSS, GHS \& ICF International (2015) is far away from the Sustainable Development Goal (SGD) 3.2 target, it is imperative that all children in Ghana receive all the doses of the measles and rota virus vaccines as well as the other vaccines.

In addition, since the Ministry of Health in Ghana confirmed the outbreak of Yellow fever in some parts of Ghana in January 2016 (Goodman, 2016) with children been one of the most vulnerable, this study sought to find out which socio-economic, geographic and demographic factors are relevant with regards to the utilization of the yellow fever and measles vaccines for children in Ghana. This would inform policy makers in charge of the Expanded Immunization Programme as well as other stakeholders on how to ensure total utilisation of these vaccines among children in Ghana. On the theoretical literature, Grossman (1999) model on the demand for health tells us individuals are the producers of health and hence the measles and the yellow fever vaccines are inputs in the health production function. Thus since children are too young to utilise theses vaccines for themselves, care givers or mothers would utilise for them in order to produce better health in these children. 
On the empirical front specifically on Ghana, Matthews and Diamond (1997), Bosu et al. (1997), Dwumoh et al. (2014), DuahOwusu (n.d.), McGlynn (2012) and Immurana and Urma (2016) have investigated factors that influence immunization utilisation among children in Ghana. However aside Immurana and Urma (2016) who investigated demand for pneumococcal and rotavirus vaccines for children in Ghana, none of these studies investigated the factors that influence the up-take of specific individual vaccines among children in Ghana. The study of the factors that affect the utilisation of individual vaccines is relevant in the sense that some mothers or care givers might utilise certain vaccines for their children but refuse to utilise other vaccines for them based on the negative perceptions they have about these vaccines. Thus to the best of the authors knowledge, this study remains the first of its kind to investigate the socio-economic, geographic and demographic factors that influence the utilisation of the two doses of the measles vaccine as well as the single dose of the yellow fever vaccine among children in Ghana.

\section{Data and methods}

The study relied on secondary data in the form of the Ghana Demographic and Health Survey which was a across-sectional survey carried out by the GHS, GSS and other partners from early September to mid-December, 2014. During the survey, information was obtained with regards to the immunization up-take among children as well other child, maternal, household and socioeconomic factors. With regards immunisation, if the date in which a vaccine was administered was found on the immunization card or if it has been ticked that a vaccine has been given to a child, or if mother could verify that a vaccine has been given to a child, this study treated any of these cases as 1 (vaccine has been utilised) and if otherwise 0 (vaccine has not been utilised). Also marital status, wealth status, and religion were recoded from their initial nature in the data.

Thus with regards to measles vaccine and yellow fever vaccine utilisation, each of the two doses of the measles vaccine and the single dose of the yellow fever vaccine were separate dependent variables and hence treated as 1 if utilised and 0 if not utilised. Since children are too young to make decisions on their own, mothers or care givers would utilise these vaccines for them based on their expected utility. Therefore following Mwabu (2008) and Strauss and Thomas (2007) as outlined in Fosu-Brefo and Arthur (2015) with some alteration, this paper assumed the utility function of a mother to be:

$$
\mathrm{U}=\mathrm{U}(\mathrm{N}, \mathrm{O}, \mathrm{H})
$$

Where $\mathrm{N}$ is a health neutral good, O refers to Socio-economic, geographic and demographic factors and $\mathrm{H}$ refers to the health status of the child.

Hence the child health production function would be:

$$
\mathrm{H}=\mathrm{F}(\mathrm{Q}, \mathrm{R},)
$$

Q refers to health inputs such as measles and yellow fever vaccines and $\mathrm{R}$ refers to the features of the child (birth order or birth rank, sex, age e.t.c). Hence the mother maximizes her utility given $\mathrm{H}$ above subject to the constraint:

$$
\mathrm{W}=\mathrm{NP}_{\mathrm{n}}+\mathrm{QP}_{\mathrm{q}}
$$

Therefore $\mathrm{W}$ refers to household Income, $\mathrm{Pn}$, and $\mathrm{P}_{\mathrm{q}}$ are the respective prices of $\mathrm{N}$, and $\mathrm{Q}$, and the health input $(\mathrm{Q})$ only enters the utility function of the mother via $\mathrm{H}$.

Manipulating equations 1, 2 and 3, we can obtain the demand function of particular vaccine as:

$\mathrm{Q}=\mathrm{D}_{\mathrm{q}}\left(\mathrm{P}_{\mathrm{q}}, \mathrm{P}_{\mathrm{n}}, \mathrm{O}, \mathrm{W}, \mathrm{R}\right)$

The above (4) which is just in a functional form can be specified simply below for the purpose of estimation as

$\mathrm{Q}=\mho \mathrm{O}+\Phi \mathrm{R}+\mu$

Q therefore becomes the probability of utilising a particular vaccine, $\mathrm{O}$ will now be Socio-economic, geographic and demographic factors such maternal education, residence type, region of residence, household wealth e.t.c. and $\mathrm{R}$ still means the features of the child such as birth order or birth rank, sex and age, $\mu$ is the error term and $\mho$ and $\Phi$ are vector of parameters of the explanatory variables. Household wealth which is now part of $\mathrm{O}$ was used to proxy household income. The price variables were omitted because vaccines are normally offered for free. Also if we were to include the price of a health neutral good, the question is which good is to be included and which should be omitted and is the good going to be common among all the respondents. Given that the dependent variables were binary, the study adopted the binary probit regression model as the empirical model of estimation. The results were reported in the form of the average marginal effects (AMEs) of the regressors in order to give the results much more intuitive meanings as Williams (2012) contends.

\section{Results and discussion}

\subsection{Descriptive statistics with Pearson chi square}

This section tackled how Measles and Yellow fever vaccines utilisation were distributed among the various categorical explanatory variables as well as Pearson chi square tests of the significance of the relationship between vaccines utilisation and the categorical explanatory variables as shown in Table 1 . From the results, it can be seen that region, religion, ethnicity, mother's education and mother's employment had statistically significant relationships with the utilisation of both the two doses of the Measles vaccine as well as the Yellow fever vaccine. Utilisation of the Measles 2 vaccine was found to have significant relationships with residence, money to seek medical care by mother, partner's education, sex of household head and household wealth status. Also the utilisation of the Measles 1 vaccine and Yellow fever vaccine were found to have significant relationships with mother's health insurance and distance to seek medical care by mother whiles sex of the child was found to have a significant relationship with only Measles 1 vaccine utilisation. Further, $41.07 \%, 36.58 \%$ and $33.19 \%$ of children from Christian, Islam and Traditional/Spiritualist/no religion backgrounds were found have received the Measles 2 vaccine respectively. Further $71.85 \%$ and $74.08 \%$ of children from nonrich and rich households respectively were found to have utilised the Yellow fever vaccine. In addition, how vaccines utilisations were distributed among all other remaining categorical independent variables can be seen in Table 1 . 
Table 1: Descriptive Statistics with Pearson Chi Square on Measles and Yellow Fever Vaccines Utilisation

\begin{tabular}{|c|c|c|c|c|c|c|c|c|c|}
\hline 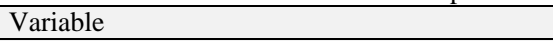 & Measl & S $1(\%)$ & Chi-square & Measl & $2(\%)$ & Chi-square & Yellov & ver $(\%)$ & Chi-square \\
\hline & No & Yes & & No & Yes & & No & Yes & \\
\hline Region & & & $55.5448 * * *$ & & & $63.5541 * * *$ & & & $58.0015 * * *$ \\
\hline Western & 28.93 & 71.07 & & 56.61 & 43.39 & & 31.73 & 68.27 & \\
\hline Greater Accra & 20.45 & 79.55 & & 59.33 & 40.67 & & 22.68 & 77.32 & \\
\hline Volta & 24.72 & 75.28 & & 57.40 & 42.60 & & 25.16 & 74.84 & \\
\hline Eastern & 25.34 & 74.66 & & 53.22 & 46.78 & & 28.57 & 71.43 & \\
\hline Ashanti & 23.38 & 76.62 & & 63.13 & 36.87 & & 26.81 & 73.19 & \\
\hline Brong Ahafo & 19.27 & 80.73 & & 56.21 & 43.79 & & 22.17 & 77.83 & \\
\hline Northern & 33.53 & 66.47 & & 70.87 & 29.13 & & 36.27 & 63.73 & \\
\hline Upper East & 23.08 & 76.92 & & 58.35 & 41.65 & & 24.16 & 75.84 & \\
\hline Upper West & 26.22 & 73.78 & & 62.79 & 37.21 & & 27.64 & 72.36 & \\
\hline Residence & & & 1.5982 & & & $4.5758 * *$ & & & 0.5709 \\
\hline Urban & 26.18 & 73.82 & & 62.24 & 37.76 & & 28.09 & 71.91 & \\
\hline Rural & 24.68 & 75.32 & & 59.37 & 40.63 & & 27.17 & 72.83 & \\
\hline Religion & & & $19.2163 * * *$ & & & $16.1092 * * *$ & & & $21.1116 * * *$ \\
\hline Christian & 24.21 & 75.79 & & 58.93 & 41.07 & & 26.55 & 73.45 & \\
\hline Traditional & 33.40 & 66.60 & & 66.81 & 33.19 & & 36.48 & 63.52 & \\
\hline Ethnicity & & & $62.0597 * * *$ & & & $42.2492 * * *$ & & & $61.7292 * * *$ \\
\hline Akan & 23.94 & 76.06 & & 57.83 & 42.17 & & 26.50 & 73.50 & \\
\hline $\mathrm{Ga} /$ Dangme & 18.95 & 81.05 & & 56.05 & 43.95 & & 21.54 & 78.46 & \\
\hline Ewe & 24.14 & 75.86 & & 59.22 & 40.78 & & 25.57 & 74.43 & \\
\hline Guan & 25.00 & 75.00 & & 60.94 & 39.06 & & 28.91 & 71.09 & \\
\hline Mole-Dagbani & 25.65 & 74.35 & & 62.41 & 37.59 & & 27.93 & 72.07 & \\
\hline Grusi & 17.80 & 82.20 & & 55.93 & 44.07 & & 18.22 & 81.78 & \\
\hline Gurma & 36.71 & 63.29 & & 71.61 & 28.39 & & 39.13 & 60.87 & \\
\hline Mande & 15.29 & 84.71 & & 61.18 & 38.82 & & 17.65 & 82.35 & \\
\hline Other & 34.29 & 65.71 & & 57.14 & 42.86 & & 35.24 & 64.76 & \\
\hline Mother's Education & & & $7.9939 * *$ & & & $21.3968 * * *$ & & & $8.0195 * *$ \\
\hline Uneducated & 27.19 & 72.81 & & 64.43 & 35.57 & & 29.75 & 70.25 & \\
\hline Primary & 25.54 & 74.46 & & 59.86 & 40.14 & & 27.22 & 72.78 & \\
\hline Secondary & 23.44 & 76.56 & & 58.14 & 41.86 & & 25.85 & 74.15 & \\
\hline Higher & 26.42 & 73.58 & & 54.25 & 45.75 & & 27.49 & 72.51 & \\
\hline Mother's Insurance & & & $10.2310 * * *$ & & & 0.5075 & & & $13.0643 * * *$ \\
\hline Marital Status & & & $3.9924 * *$ & & & 0.3975 & & & $3.1917 *$ \\
\hline Single & 26.92 & 73.08 & & 61.10 & 38.90 & & 29.05 & 70.95 & \\
\hline Married & 24.45 & 75.55 & & 60.22 & 39.78 & & 26.78 & 73.22 & \\
\hline Mother's Employment & & & $65.6118 * * *$ & & & $25.1398 * * *$ & & & $59.9344 * * *$ \\
\hline Unemployed & 34.52 & 65.48 & & 66.96 & 33.04 & & 36.63 & 63.37 & \\
\hline Employed & 22.88 & 77.12 & & 58.86 & 41.14 & & 25.19 & 74.81 & \\
\hline Permission to seek medical care/help by mother & & & 1.2783 & & & 0.3115 & & & 0.1057 \\
\hline Big Problem & 27.73 & 72.27 & & 61.87 & 38.13 & & 28.27 & 71.73 & \\
\hline Not a Big Problem & 25.11 & 74.89 & & 60.41 & 39.59 & & 27.49 & 72.51 & \\
\hline Money to seek medical care/help by mother & & & 1.7387 & & & $6.5737 *$ & & & 1.8186 \\
\hline Big Problem & 26.08 & 73.92 & & 62.24 & 37.76 & & 28.38 & 71.62 & \\
\hline Not a Big Problem & 24.54 & 75.46 & & 58.88 & 41.12 & & 26.76 & 73.24 & \\
\hline Distance to seek medical care/help by mother & & & $4.7203 * *$ & & & 2.4377 & & & $5.2220 * *$ \\
\hline Big Problem & 27.14 & 72.86 & & 62.01 & 37.99 & & 29.55 & 70.45 & \\
\hline Not a Big Problem & 24.42 & 75.58 & & 59.81 & 40.19 & & 26.61 & 73.39 & \\
\hline Partner's Education & & & 3.7689 & & & $13.0635^{* * *}$ & & & 5.3803 \\
\hline Uneducated & 26.39 & 73.61 & & 63.73 & 36.27 & & 28.95 & 71.05 & \\
\hline Primary & 25.08 & 74.92 & & 57.51 & 42.49 & & 27.80 & 72.20 & \\
\hline Secondary & 23.70 & 76.30 & & 59.28 & 40.72 & & 25.68 & 74.32 & \\
\hline Higher & 25.00 & 75.00 & & 56.90 & 43.10 & & 27.00 & 73.00 & \\
\hline Sex of Household Head & & & 0.2324 & & & $3.6960 *$ & & & 0.0068 \\
\hline Male & 25.43 & 74.57 & & 61.21 & 38.79 & & 27.56 & 72.44 & \\
\hline Female & 24.77 & 75.23 & & 58.23 & 41.77 & & 27.45 & 72.55 & \\
\hline Sex of Child & & & $2.9739 *$ & & & 0.1457 & & & 2.3170 \\
\hline Male & 26.24 & 73.76 & & 60.75 & 39.25 & & 28.41 & 71.59 & \\
\hline Female & 24.24 & 75.76 & & 60.25 & 39.75 & & 26.59 & 73.41 & \\
\hline Wealth Status & & & 1.8147 & & & $3.9217 * *$ & & & $2.7735 *$ \\
\hline Non-Rich & 25.76 & 74.24 & & 61.31 & 38.69 & & 28.15 & 71.85 & \\
\hline Rich & 24.00 & 76.00 & & 58.40 & 41.60 & & 25.92 & 74.08 & \\
\hline
\end{tabular}

Source: Authors computation from the 2014 GDHS. Notes: $1 . * * *$, and $* * *$ showing significant difference at $10 \%, 5 \%$ and $1 \%$ respectively within the predictor and measles and yellow fever vaccines utilisation among children in Ghana. 2 . Traditional in this study means traditional/ spiritualist/no religion. 
Table 2: Probit Regressions on Determinants of Demand for Measles and Yellow Fever Vaccines

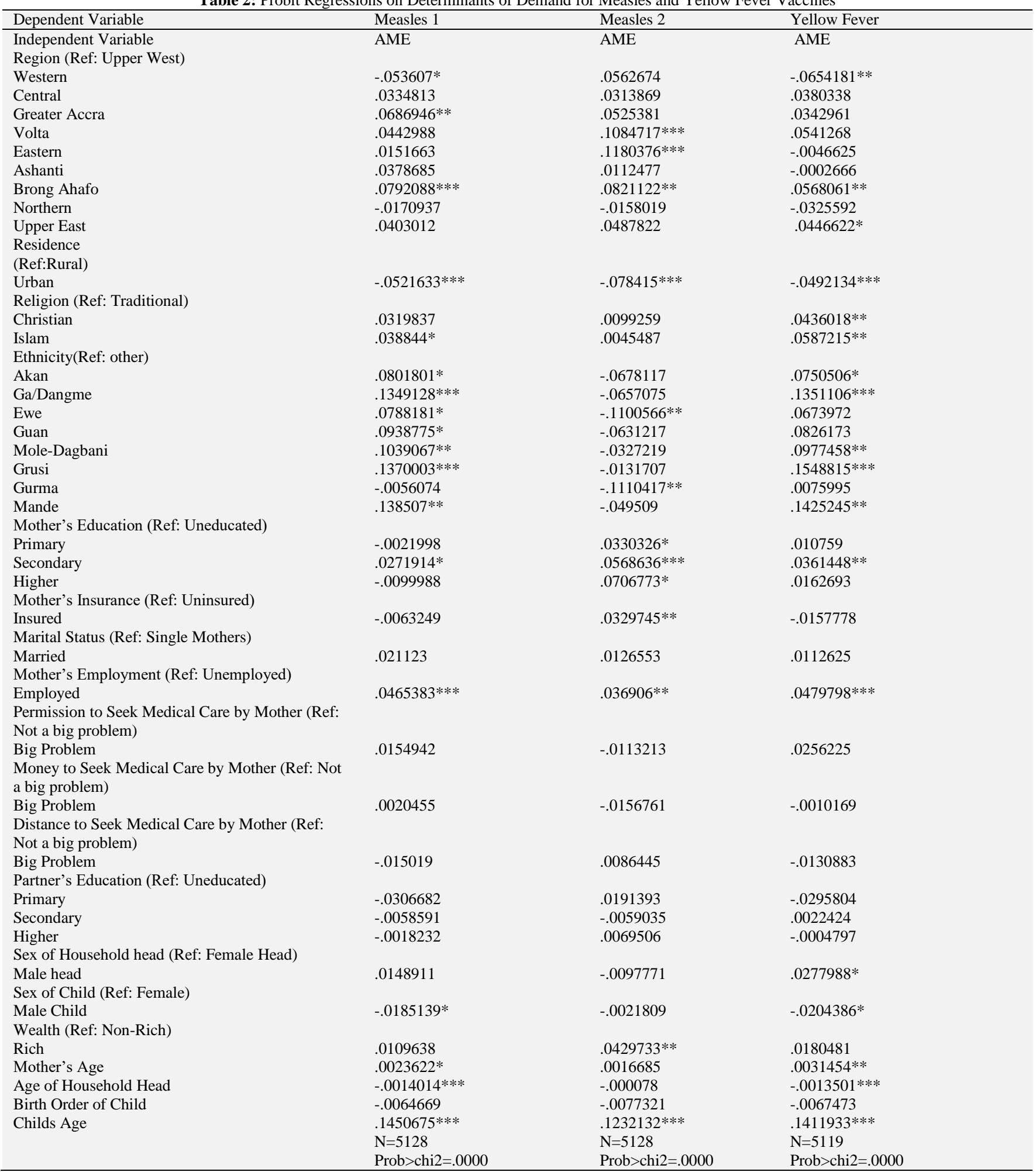

Source: Authors computation from the 2014 GDHS. Notes: 1 . ***P-value<.01, **P-value<.05, *P-value<.1 2. Traditional in this study means traditional/ spiritualist/no religion.

\subsection{Multivariate analyses}

From the results using the AMEs, children from the Western, Brong Ahafo and Greater Accra regions were 5\% less likely, $8 \%$ and $7 \%$ more likely to have received the Measles 1 vaccine respectively relative to those in the Upper west region. The results on the Western region on one hand and those on Brong Ahafo and Greater Accra regions one another hand conflicts and concur re- spectively with the findings of Immurana and Urma (2016) in the case of demand for pneumococcal vaccine for children in Ghana. Also, urban children were revealed to be $5 \%$ less likely to receive the Measles 1 vaccine as compared with rural children. This could be attributed to probably the overconcentration of child health utilisation drives on rural areas to the neglect of urban areas given that rural dwellers are normally seen to be less advantaged. This results together with that on region, tell us the importance of geographic area of residence on the likelihood of utilising vaccines among children in Ghana. Further, Muslim children were found to 
be $4 \%$ more likely to receive the Measles 1 vaccine relative to Traditional/Spiritualist/No Religion faiths children.

In addition, Ga/Dangme, Akan, Guan, Ewe, Mole-Dagbani, Mande and Grusi ethnicity children were on the average 13\%, $8 \%$, $9 \%, 8 \%, 10 \% 14 \%$ and $14 \%$ respectively more probable to have received the Measles 1 vaccine relative to those from the other ethnic backgrounds (reference group).

On the average, mothers with secondary education were found to be 3\% more likely to demand the Measles 1 vaccine for their children as compared with the uneducated mothers. This is not surprising since the educated have higher ability to understand the essence of health inputs such as vaccines towards child health or survival and hence would utilise them more for their children relative to the uneducated mothers.

Also employed mothers were revealed on average to be $5 \%$ more likely to demand the Measles 1 vaccine for their children as compared with the unemployed mothers. This is not surprising since even though these vaccines are normally offered for free, there could be indirect costs such as transportation cost to immunisation centres in which employed mothers can afford better than their unemployed counterparts. Also it could be that employed mothers value so much the time they would waste on seeking treatment for their children when they get sick and hence would adopt more preventive approaches such as vaccine utilisation. Similar results were found by Immurana and Urma (2016) who revealed employed mothers to be more likely to demand for all the individual doses of the pneumococcal and rotavirus vaccines for children in Ghana.

Further, male children were found to be $2 \%$ less likely to receive the Measles 1 vaccine relative to female children. This can be attributed to the well-grounded believe that boys are relatively stronger than girls and hence the tendency of utilising these vaccines more for female children relative to male children.

Also, on average, a yearly increase in the ages of the child and mother were found to increase the probability that a child received the Measles 1 vaccine respectively by $15 \%$ and $.2 \%$. This is similar to the result of Otieno et al. (2014) who revealed older maternal and child age to be linked with the probability that a child received influenza vaccination in Kenya. The results on rising mother's age and rising Measles 1 vaccine utilisation could be that mothers (when they were younger) might have had some negative experiences of not utilising these vaccines for their children and hence would be willing to utilise for their later births as they grow older (mothers). Thus older mothers may appreciate the essence of these vaccines to child health relative to younger mothers. Conversely on average, a yearly increase in the age of the household head was found to decrease the likelihood that a child received the Measles 1 vaccine by .1\%. This could be attributed to the unwillingness on the part of some elderly persons in the Ghanaian society to accept modern medicines believing that, they (modern medicines) have lots of bad side effects.

On demand for Measles 2 vaccine, the results showed that children from the Volta, Brong Ahafo and Eastern regions were $11 \%$, $8 \%$ and $12 \%$ respectively more probable to have received the Measles 2 vaccine relative to those in the Upper west region. This conflicts that of Logullo et al. (2008) who revealed region not to be associated with adequate up-take of measles vaccine in Sao Paulo City (Brazil). Also on average, urban children were found to be $8 \%$ less likely to receive the Measles 2 vaccine relative to rural children. This is synonymous to the results on Measles 1 and hence further reinforces the likelihood that less attention is given to urban areas in favour of rural areas in child health utilisation drives believing that urban children are less vulnerable relative to rural children. In addition, children of Gurma and Ewe ethnicities were both $11 \%$ less probable to have received the Measles 2 vaccine relative to those in the other ethnic groups. As expected, mothers with Primary, Higher and Secondary levels of education were $3 \%, 7 \%$ and $6 \%$ more likely to demand the Measles 2 vaccine for their children relative to the uneducated mothers. Thus mother's education positively influenced the demand for Measles 2 vaccine. This is in line with the results of Cockcroft et al. (2009) and Cockcroft et al. (2014) who revealed mother's education to influence the probability that a child received the measles vaccine in Pakistan and Nigeria respectively. Also on average mothers with health insurance and employment were respectively $3 \%$ and $4 \%$ more likely to demand the Measles 2 vaccine for their children relative to mothers with no health insurance and unemployed mothers respectively. This is not surprising because child health immunisation drives are also carried out at health facilities and since mothers with health insurance are more likely to access health facilities relative to those without health insurance, they may end-up utilising vaccines more for their children relative to their counterparts without health insurance. The above explanation becomes more valid in the Ghanaian set-up where the National Health Insurance Scheme with the highest number of enrolees has a free maternal and child health insurance registration scheme. Further, wealthy households were $4 \%$ more probable to demand the Measles 2 vaccine for their children as compared with the nonwealthy households. This concurs with the results of Cockcroft et al. (2009) who found children from less vulnerable households to be more probable to receive the measles vaccine in Pakistan. This could be that although most of these vaccines are offered for free, wealthy households are well empowered to afford indirect costs of immunisation such as transportation cost as compared to nonwealthy households. Also a yearly increase in child's age was found to increase the probability of demanding the Measles 2 vaccine for children by $12 \%$. This is similar to the results of Otieno et al. (2014) who found older children to be associated with the likelihood of receiving influenza vaccination in Kenya. The rising child's age that has been found to increase the likelihood of demanding both the Measles 1 and 2 doses could be that, since these doses are offered at ages nine months and eighteen months respectively when children are relatively older, older children are more likely to receive relative to younger children.

On the Yellow fever vaccine, on average the results showed that children in the Western, Upper East and Brong Ahafo regions were respectively $7 \%$ less likely, $4 \%$ and $6 \%$ more likely to have received the Yellow fever vaccine relative to those in the Upper West region.

Also on average, urban children were 5\% less likely to have received the Yellow fever vaccine as compared with rural children. This is similar to the findings under both Measles 1 and 2 vaccines and hence confirming the possible neglect of urban areas to the advantage of rural children in child health utilisation drives.

Also Christian and Muslim children were respectively $4 \%$ and $6 \%$ more likely to have received the Yellow fever vaccine relative to Traditional/Spiritualist/No religion faiths children. This could be attributed to the believes of some Traditional faith people that traditional medicine is part of the traditions bequeathed to them by their forefathers and hence would not throw it away for "alien medicines" such as the Yellow fever vaccine.

Moreover, Ga/Dangme, Akan, Mole-Dagbani, Mande and Grusi children were $14 \%, 8 \%, 10 \%, 14 \%$ and $15 \%$ respectively more likely to have received the Yellow fever vaccine as compared with those from the other ethnic groups. Further, mothers with Secondary level of education and employed mothers were $4 \%$ and $5 \%$ respectively more likely to demand the Yellow fever vaccine for their children relative to the uneducated and unemployed mothers. In addition, children with male household heads and male children were $3 \%$ more likely and $2 \%$ less likely to have received the Yellow fever vaccine relative to those with female heads and female children respectively. The results on male household heads conflicts the findings of Immurana and Urma (2016) who revealed male household heads to be less likely to demand for rotavirus vaccine for children in Ghana.

Also on average, as the ages of the child and mother increased by a year, the probability that a child received the Yellow fever vaccine increased by $14 \%$ and $.3 \%$ respectively. The finding on child's age conflicts the findings of Immurana and Urma (2016) who found rising child's age to be associated with the less likelihood to demand for rotavirus and pneumococcal vaccines for children in Ghana. The findings showing rising child's age increased 
the utilisation of the Yellow fever vaccine could be that, since the Yellow fever vaccine is offered at age nine months similar to the measles 1 vaccine when children are relatively older, older children are more likely to receive relative to younger children.

Last but not the least, on average, a yearly increase in the age of the household head decreased by .1\%, the probability that a child received the Yellow fever vaccine. Similar result was observed under Measles 1 and hence the explanation applies. These findings are similar to the results of Immurana and Urma (2016) who revealed rising age of the household head to decrease the demand for pneumococcal 3 vaccine for children in Ghana.

\section{Conclusion}

Based on the findings, the study concludes that residence, region, ethnicity, child's age, mother's education and employment are the factors that affect the demand for the Measles vaccine among children in Ghana irrespective of the dose number. Also household wealth status, residence, region, ethnicity, mother's health insurance, education and employment and Child's age are the factors that determine the demand for the subsequent dose of the Measles vaccine among children in Ghana. In addition, ethnicity, residence, region, religion, mother's age, education and employment, sex and age of the child, sex and age of the household head are the determinants of demand for the Yellow fever vaccine among children in Ghana. Thus in all a whole, the general conclusion is that Socio-Economic, Demographic and Geographic Factors are relevant determinants of demand for measles and yellow fever vaccines among children in Ghana.

Therefore aiding uneducated, younger and unemployed mothers, aiding poor households, reinvigorating the behaviour change communication with regional and ethnic dimensions, demystifying the irrelevance of child's sex in health utilisation, reducing the possible over concentration in rural areas and further encouraging maternal health insurance registration as short term policies as well as well encouraging girl child education, reducing poverty and reducing women unemployment as long term policies, could be effective tools in encouraging child health utilisation (utilising Measles and Yellow fever vaccines) in Ghana.

\section{Acknowledgement}

We are thankful to the DHS Program for the data.

\section{Conflicts of interest}

None declared

\section{References}

[1] Bosu KW, Ahelegbe D, Edum-Fotwe E, Bainson AK \& Turkson KP (1997) Factors influencing attendance to immunization sessions for children in a rural district of Ghana. Acta Tropica 68, 259-267, Elsevier. http://dx.doi.org/10.1016/S0001-706X(97)00094-6.

[2] Cockcroft A, Usman UM, Nyamucherera FO, Emory H, Duke B Umar AN \& Andersson N (2014) Why children are not vaccinated against measles: a cross-sectional study in two Nigerian States. Archives of Public Health, 72(48). Retrieved from http://www.archpublichealth.com/content/72/1/48. http://dx.doi.org/10.1186/2049-3258-72-48.

[3] Cockcroft A, Andersson N, Omeri K, Ansari MN, Khan A, Chaudhry UU, \& Ansari U (2009) One size does not fit all: local determinants of measles vaccination in four districts of Pakistan. BMC International Health and Human Rights, 9,(Suppl 1):S4 http://dx.doi.org/10.1186/1472-698X-9-S1-S4.

[4] Duah-Owusu M (n.d.) Social Determinants and Immunisation in Ghana: Is there an Association? Unpublished MPhil Thesis, University of Bergen research centre for health promotion and development, faculty of psychology.
[5] Dwumoh D, Essuman EE \& Afagbedzi KS (2014) Determinant of factors associated with child health outcomes and service utilization in Ghana: multiple indicator cluster survey conducted in 2011. Archives of Public Health, 72(42). Retrieved from http://www.archpublichealth.com/content/72/1/42. http://dx.doi.org/10.1186/2049-3258-72-42.

[6] Fosu-Brefo, R. \& Arthur, E. (2015) Effect of timely initiation of breastfeeding on child health in Ghana. Health Economics Review, 5(8). http://dx.doi.org/10.1186/s13561-015-0044-8.

[7] Ghana Statistical Service (GSS), Ghana Health Service (GHS), \& ICF International. Ghana Demographic and Health Survey 2014. Rockville, Maryland, USA: GSS, GHS, and ICF International, 2015.

[8] Goodman, T (2016). Outbreak of Meningitis and Yellow Fever in some Parts of the Country. Accessed from: http://citifmonline.com/2016/01/07/health-ministry-confirmsoutbreak-of-meningitis-yellow-fever-in-ba/ on Thursday 7th January, $20165: 26 \mathrm{pm}$.

[9] Grossman M (1999) the Human Capital Model and the Demand for Health. NBER Working Paper No. 7078.

[10] Immurana M, \& Urma, A (2016) Determinants of demand for subsequent doses of pneumococcal and rotavirus vaccines for children less than five years of age in Ghana. International Journal of Health, 4 (2). doi: 10.14419/ijh.v4i2.6114

[11] Logullo P, de Carvalho BH, Saconi R, \& Massad E (2008) Factors affecting compliance with the measles vaccination schedule in a Brazilian city, Sao Paulo. Sao Paulo’ Med J., 126(3),166-71.

[12] Matthews Z \& Diamond I (1997) Child Immunisation in Ghana: The Effects of Family, Location and Social Disparity, Journal of Biosocial Science, 29(03), 327 - 343. DOI: null, Published online: 04 April 2001.

[13] McGlynn KN (2012) Antenatal Care as a Determinant of Immunization, and Appropriate Care for Fever and Diarrhoea in Ghanaian Children. Unpublished MSc Thesis in Epidemiology \& Biostatistics, the School of Graduate and Postdoctoral Studies, the University of Western Ontario, London, Ontario, Canada.

[14] Mwabu G. The Production of Child Health in Kenya: A Structural Model of Birth Weight. Economic growth center Yale University, New Haven, Center discussion paper 2008 no. 963.

[15] Otieno AN, Nyawanda OB, Audi A, Emukule G, Lebo E, Bigogo G, Ochola R, Muthoka P, Widdowson M-A, Shay KD, Burton CD, Breiman FR, Katz AM \& Mott AJ (2014) Demographic, socioeconomic and geographic determinants of seasonal influenza vaccine uptake in rural western Kenya, 2011. Vaccine 32, 6699-6704. Retrieved from http://dx.doi.org/10.1016/j.vaccine.2013.10.089.

[16] Strauss J \& Thomas D (2007) Health over the life course. Handbook of development economics, 4:3474-375. http://dx.doi.org/10.1016/S1573-4471(07)04054-5.

[17] Williams R. (2012). Using the margins command to estimate and interpret adjusted predictions and marginal effects. The Stata Journal, 12(2), 308-331.

[18] World Health Organisation (2016a). Measles: Fact Sheet reviewed March, 2016. Accessed from: http://www.who.int/mediacentre/factsheets/fs286/en/ on 02/06/2016.

[19] World Health Organisation (2016b). Yellow fever: Fact Sheet updated May, 2016. Accessed from: http://www.who.int/mediacentre/factsheets/fs100/en/ on 02/06/2016.

[20] World Health Organisation. (2014). Yellow Fever, Fact Sheet $N^{0}$ 100. March, 2014. 